\title{
Multiplex Immunoassay of Cytokines for The Short- Term Prognosis Predictive Value of Patients With Acute-on-Chronic Liver Failure
}

\section{Yongyuan Zheng}

Department of Infectious Diseases

\section{Genglin Zhang}

Department of Infectious Diseases

\section{Lina Wu}

Department of Infectious Diseases

\section{Jing Xiong}

Department of Infectious Diseases

\section{Lu Wang}

Department of Infectious Diseases

\section{Xuejun Li}

Department of Infectious Diseases

Jianguo Li

Department of Infectious Diseases

\section{Chan Xie}

Department of Infectious Diseases

Liang Peng ( $\square$ pliang@mail.sysu.edu.cn )

Third Affiliated Hospital of Sun Yat-Sen University https://orcid.org/0000-0001-6184-5750

\section{Research}

Keywords: luminex, liver failure, prognosis, IL-6

Posted Date: January 29th, 2021

DOl: https://doi.org/10.21203/rs.3.rs-154337/v1

License: (c) (i) This work is licensed under a Creative Commons Attribution 4.0 International License. Read Full License 


\section{Abstract}

Background: Since the systemic inflammation has been found to be associated with disease progression and mortality in patients with hepatitis B virus (HBV)-related acute-on-chronic liver failure (HBV-ACLF), the objective of this study was to detect inflammatory factors in ACLF patients by a Luminex-based multiplex immunoassay system for high throughput screening of the cytokine with the most prognostic value.

Methods: Luminex-based multiplex immunoassay technology was used to determine the concentrations of 48 cytokines in total at once in serum samples from 40 patients with HBV-ACLF, 30 patients with chronic hepatitis $B(\mathrm{CHB})$ and 25 healthy volunteers as normal controls $(\mathrm{NC})$. Then, the receiver operating characteristic (ROC) curve analysis was applied to evaluate the prognostic prediction accuracy. Besides, Kaplan-Meier curves was used to analyze survival, while the Cox regression analysis to determine the mortality predictors.

Results: The level of IL-6, IL-10, IL-15, IL-18, M-CSF, IP-10 and CXCL9 were significantly higher in patients with HBV-ACLF than in either patients with CHB or NC subjects, while the level of EGF, PDGF-AA, PDGF$A B / B B, M D C$ and SCD40L were significantly lower. The concentrations of IL-6, CXCL9, and IL-15 was higher in non-surviving patients with HBV-ACLF than in surviving patients while MDC was lower. Increased serum IL- 6 was positively correlated with disease severity. The ROC curve analysis showed that IL-6 and CXCL-9 accurately predicted 90-day survival in patients with HBV-ACLF, with an accuracy equivalent to those of the Model for End-Stage Liver Disease (MELD), MELD-Na. Kaplan-Meier analysis showed an association between the increase in serum concentration of IL- 6 as well as CXCL9 and poor overall survival in patients with HBV-ACLF. Moreover, the multivariate Cox regression analysis showed that only serum IL-6 was an independent predictor of overall survival in patients with HBV-ACLF.

Conclusion: Although HBV-related ACLF patients have significantly increased serum levels of multiple cytokines, only serum IL-6 levels could be an independent prognostic biomarker in patients with HBVACLF.

\section{Introduction}

Acute-on-chronic liver failure (ACLF) is a distinct clinical syndrome characterized by liver failure due to acute hepatic injury and underlying chronic liver disease with high 28-day mortality. The pathogenesis of hepatitis B virus-related acute-on-chronic liver failure (HBV-ACLF) is complicated and considered to be caused by the superposition of multiple factors, such as the reactivation of HBV, bacterial and fungal infection, abnormal immune cell function and distribution, systemic inflammatory response (SIRS), and immune paralysis[1]. Accumulating evidence has shown that systemic inflammation caused by excessive immune-mediated inflammation plays a central role in the mechanism underlying HBV-ACLF. Moreover, systemic inflammation has been found to be associated with disease progression and mortality in patients with HBV-ACLF [2-5]. Thus, many studies tried to test some inflammatory factors when patients were admitted to the hospital, combined with the research background of these factors, hoping to screen 
for factors with prognostic value, and even served as potential drug targets. In fact, cytokines such as IL10, IL-22, IL-27, Fractalkine, interferon gamma-induced protein 10 (IP-10) [6-9] have been reported to have certain predictive value in ACLF diseases. However, these cytokines are rarely used in clinical practice so far, probably because the predictive value of these factors is not consistent in different studies. Therefore, we used luminex multiple analyte profiling technology to determine the concentrations of over 40 cytokines in serum samples from ACLF patients at once, to screen out the inflammatory factors with the most prognostic value.

\section{Methods}

\section{Study design and patients}

Forty patients with HBV-ACLF admitted to our department between May 2018 and June 2020 were enrolled. HBV-ACLF was diagnosed on the basis of the development of jaundice (a TBil of $\leq 171 \mu \mathrm{mol} / \mathrm{L}$ ), a INR of $\geq 1.5$, and the presence of at least one of the other criteria ( $\geq$ grade 2 hepatic encephalopathy, ascites, spontaneous bacterial peritonitis, or hepatorenal syndrome). The exclusion criteria were: 1) evidence of other liver diseases or cancer; 2) coinfection with other hepatitis viruses or HIV; 3) treatment with artificial liver support or immunomodulatory drugs; 4) drug or alcohol abuse; 5) history of cardiovascular, pulmonary, or renal diseases; and 6) pregnancy. Cirrhosis was clinically diagnosed when a small, nodular liver was observed in imaging tests, including ultrasound, computerized tomography scans, or magnetic resonance imaging. Each patient was treated with supportive internal treatment. All patients with HBV-ACLF were followed up for at least 3 months. The patients' outcomes were recorded as surviving or non-surviving. Thirty patients with chronic hepatitis $B(\mathrm{CHB})$ and twenty-five healthy volunteers as normal controls (NC) during the same period were enrolled as controls. Patients with $\mathrm{CHB}$ were defined as those who had showed HBsAg positivity for more than 6 months and exhibited signs of hepatitis and abnormal liver function. A clinical assessment was performed and peripheral blood was collected at admission. Serum was separated and stored at $-80^{\circ} \mathrm{C}$ until analysis. The study was conducted in accordance with the guidelines of the Declaration of Helsinki, and the protocol was approved by the ethics committee of our hospital (The Third Affiliated Hospital of Sun Yat-sen University). Written informed consent was obtained from each participant.

\section{Luminex for serum cytokine measurements.}

Serum samples from ACLF, CHB and HC were used to analyze the concentrations of 48 cytokines in duplicate using Human Cytokine Antibody-Immobilized Magnetic Beads (Millipore, Billerica, Massachusetts). The cytokines were as follows: interleukin-1 alpha (IL-1a), IL-1b, IL-1RA, IL-2, IL-3, IL-4, IL5, IL-6, IL-7, IL-8, IL-9, IL-10, IL-12p40, IL-12p70, IL-13, IL-15, IL-17, IL-18, IL-22, IL-27, epidermal growth factor (EGF), eotaxin, granulocyte colony-stimulating factor (G-CSF), granulocyte macrophage colonystimulating factor (GM-CSF), interferon alpha-2 (IFN-a2), interferon gamma-induced protein 10 (IP-10), IFNy, monocyte chemotactic protein-1 (MCP-1), MCP-3, MIP-1a, MIP-1b, tumor necrosis factor alpha (TNFa), TNF $\beta$, vascular endothelial growth factor (VEGF), fibroblast growth factor 2 (FGF-2), transforming 
growth factor alpha (TGF-a), Flt-3 ligand (FIT-3L), fractalkine, growth-regulated oncogene (GRO), macrophage-derived chemokine (MDC), soluble CD40 ligand (SCD40L), chemokine (C-X-C motif) ligand 9 (CXCL9). Measurements were performed on the Luminex-MAGPIX multiplex immunoassay system. Data were analyzed using Milliplex Analyst 5.1 software (EMD Millipore, Billerica, Massachusetts).

\section{Virological assessment and liver biochemical assays}

The levels of serum HBeAg was measured using an Elecsys system (Hoffman-La Roche Ltd., Basel, Switzerland). HBV-DNA levels were quantitated with RT-PCR using an ABI7300 instrument (Thermo Fisher Scientific). The detection limit of HBV -DNA was $100 \mathrm{IU} / \mathrm{ml}$. Biochemical assays were performed using an auto-analyzer (TBA-30FR; Toshiba, Tokyo, Japan). PTA was measured using an automatic hemostasis/thrombosis analyzer (STA Compact, Holliston, MA, USA).

\section{Disease severity assessment}

MELD, MELD-Na were used to assess disease severity. Briefly, these scores were calculated as follows: MELD score $=3.8 \times \ln ($ bilirubin $[\mathrm{mg} / \mathrm{dL}])+11.2 \times \ln (\mathrm{INR})+9.6 \times \ln ($ creatinine $[\mathrm{mg} / \mathrm{dL}])+6.4 \times($ etiology: 0 if cholestatic or alcoholic, 1 otherwise); MELD-Na score=MELD score-Na-0.025 $\times$ MELD $\times(140-\mathrm{Na})+140$.

\section{Statistical analyses}

Data were analyzed using SPSS version 22.0 (IBM Corporation, Armonk, NY, USA) and were expressed as frequencies, medians, and ranges or mean \pm standard deviations. Differences in variables were analyzed with ANOVA and Student's t-tests or with Kruskal-Wallis tests and Mann-Whitney U tests. Correlations were evaluated by Pearson or Spearman tests. ROC curves were used to predict prognosis. Comparisons of ROC curve parameters were performed using the DeLong test. Survival was analyzed using KaplanMeier curves. The association between relevant variables and mortality was investigated by the multivariate Cox regression analysis. Two-sided P-values of $<0.05$ were considered statistically significant.

\section{Results}

\section{Patients' characteristics}

The median age of the patients with HBV-ACLF was about 43 years. During the follow-up period, 25 patients with HBV-ACLF survived, while 15 died. Thus, the overall mortality rate was $37.5 \%$. Twenty-nine (72.5\%) patients with HBV-ACLF were clinically diagnosed with cirrhosis before enrollment. The baseline characteristics of the participants are shown in Table 1. No significant differences existed among the three groups in age $(P=0.170)$ or gender $(P=0.877)$.

\section{Luminex detected the serum level of forty-eight inflammatory factors in patients with HBV-ACLF}


We measured the serum concentrations of 48 cytokines by luminex among three groups. (Figure $1 \mathrm{~A}$ ). The level of IL-6, IL-10, IL-15, IL-18, M-CSF, IP-10 and CXCL9 were significantly higher in patients with HBVACLF than in either patients with CHB or NC subjects. While the level of EGF, PDGF-AA, PDGF-AB/BB, MDC and SCD40L were significantly lower in patients with HBV-ACLF than other groups. (Figure $1 \mathrm{~B}$ )

We then determined the correlation between clinical outcome and these 12 cytokines and found four cytokines with predictive value including IL-6, IL-15, CXCL9 and MDC.

\section{IL-6 correlated with disease severity in HBV-ACLF}

The serum level of IL-6 was significantly higher in non-surviving patients (median $14.41 \mathrm{pg} / \mathrm{ml}$, range $1.07-114.91 \mathrm{pg} / \mathrm{ml}$ ) than in surviving patients (median $6.98 \mathrm{pg} / \mathrm{ml}$, range $1.53-15.10 \mathrm{pg} / \mathrm{ml}, \mathrm{P}<0.01$; Figure $2 \mathrm{~A}$ ). While the serum levels of IL-15, CXCL9 and MDC were statistically different between surviving groups and non-surviving groups. We subsequently analyzed the correlation between the serum levels of these cytokines and PTA levels, INR levels and disease severity parameter like MELD and MELD-Na scores. Interestingly, positive correlations were found between IL- 6 serum level and MELD score $(r=0.359$, $P=0.0234)$, MELD-Na score $(r=0.365, P=0.0204$; Figure $2 A)$. In addition, MDC serum level had negative correlation with MELD-Na score ( $r=-0.386, P=0.014$; Figure 2C). Besides, CXCL9 serum level seem to have no correlation with MELD and MELD-Na score, while correlated with PTA ( $r=-0.365, P=0.022)$ and INR $(r=-0.395, P=0.012$; Figure $2 \mathrm{D})$. However, IL-15 have no correlation with these parameters. Collectively, these findings indicated IL-6, MDC, CXCL9 may have more predictive value.

\section{Increased serum level of IL-6 and CXCL9 indicated poor prognosis in patients with HBV-ACLF}

The ROC curve analysis was used to evaluate the value of four cytokines mentioned earlier in predicting prognosis (Figure 3A). In addition, the ES index was used to identify the optimal cutoff value, defined as the value that maximized the sensitivity and specificity. The ROC curve analysis results were shown in Table 2. The area under the ROC curve (AUROC) of IL- 6 was 0.703 (95\% Cl: $0.507-0.899, P=0.034)$, CXCL9 was 0.691 (95\% Cl: $0.510-0.871, P=0.046)$. Compared with the MELD score (AUROC=0.765, $P=0.005$ ), MELD-Na score (AUROC=0.779, $\mathrm{P}=0.004)$, IL-6 and CXCL9 still had a promising prognostic value.

Patients were then divided into two groups by each cut-off value, including IL-6, CXCL9, MELD, MELD-Na. The 90-day survival rate was examined by the Kaplan-Meier analysis between higher group and lower group. The log-rank test revealed significant differences between the higher group and the lower group in 90-day survival rate (IL-6, $P=0.032 ; C X C L 9, P=0.012 ;$ MELD, $P=0.001$; MELD-Na, $P<0.001$; Figure 3B). Collectively, these data suggested that an increased frequency of IL-6 and CXCL9 could be useful predictors of mortality in patients with HBV-ACLF.

\section{Increased serum level of IL-6 could be an independent predictor of mortality}

Baseline clinical and laboratory variables were analyzed as possible predictors of mortality. The basic characteristics of surviving and non-surviving patients with HBV-ACLF are summarized in Table 3. Compared with surviving patients, non-surviving patients were had higher levels of WBC, INR, Cr and PCT, 
had lower levels of PLT and $\mathrm{Na}$ (Table 3). Next, the Cox regression analysis was used to identify predictors for HBV-ACLF. In the univariate analysis, the serum level of IL-6, IL-15, CXCL9, WBC, PLT, INR, $\mathrm{Na}, \mathrm{CR}, \mathrm{PCT}, \mathrm{MELD}$ score, MELD-Na score were factors associated with a higher risk of mortality (Table 4). Next, we evaluated these significant variables in a multivariate Cox regression analysis by using forward stepwise (likelihood ratio) selection. Only IL-6 (HR=1.026, $P=0.025), W B C(H R=1.344, P<0.001)$, and PLT (HR=0.979, $P=0.009)$ were found to be independent baseline predictors of mortality in patients with HBV-ACLF (Table 4).

\section{Discussion}

In this study, we used luminex to detect the levels of more than 40 inflammatory factors in the serum of ACLF patients in large quantities at one time, since systemic inflammatory response (SIRS) played an important role in the pathogenesis and disease progression of ACLF. First, we compared with CHB group and $\mathrm{HC}$ group to figure out more specific inflammatory factors of $\mathrm{ACLF}$, and then further analyzed with the prognostic outcome to screen out the cytokines with the most prognostic value. As a matter of fact, Wang[6] have used cytometric bead array (CBA) to test Interleukin-1 $\beta$ (IL-1 $\beta$ ), IL-2, IL-4, IL-6, IL-8, IL-10, IL12 p70, TNF- $\alpha$ and IFN-y protein levels in blood plasma between patients with acute exacerbation of chronic hepatitis B (CHB) and patients with HBV-related acute-on-chronic liver failure (HBV-ACLF). They found that assessment of plasma IL-10 levels in CHB acute exacerbation might provide an early predictive marker for progression to HBV-ACLF. Meanwhile, Cristina Solé[8] also measured a large array of plasma cytokines between patients with cirrhosis and with ACLF. They found that cytokines showing the most marked relationship with ACLF were VCAM-1 and VEGF-A. In the researches mentioned above, there were actually many overlaps of cytokines tested including IL-6, compared with our study. However, the differences including enrollment criteria, the tested specimens, even the research purposes resulted in incomplete conclusions. While in this study, among the 48 inflammatory factors we detected, only IL-6 is shown to be an independent prognostic factor by the multivariate Cox regression analysis.

Interleukin 6 (IL-6) is a pleiotropic four-helix-bundle cytokine that exerts multiple functions in the body. In the liver, IL-6 is an important inducer of the acute phase response and infection defense. IL- 6 is furthermore crucial for hepatocyte homeostasis and is a potent hepatocyte mitogen. It is not only implicated in liver regeneration, but also in metabolic function of the liver. However, persistent activation of the IL- 6 signaling pathway is detrimental to the liver and might ultimately result in the development of liver tumors [10,11]. Although IL-6 has been considered to have predictive value in many diseases [12-16], the role and the prognostic value of IL- 6 in patients with HBV-ACLF has never been reported. As we know, IL-6 is a multifunctional cytokine that transmits cell signaling and regulates immune cells. This factor has a strong proinflammatory effect with multiple biological functions and plays an important role in inflammation, tumor, and hematological diseases $[17,18]$. IL- 6 is the primary trigger for cytokine storms. Dong Wook Jekarl [16] used the biomarkers PCT, IL-6, C-reactive protein (CRP) to evaluate the diagnosis and prognosis of sepsis after antimicrobial therapy among systemic inflammatory response syndrome (SIRS), and indicated IL-6 as a prognostic marker for sepsis. Studies have shown that IL-6 cannot only be used as an early diagnostic biomarker of bacterial infection in patients with liver cirrhosis [14], but also is 
related to the severity of liver cirrhosis. Patients with liver cirrhosis with elevated IL-6 have a greater risk of hepatic encephalopathy complications. In addition, it is more likely to develop liver failure $[15,19]$. However, IL- 6 can be used as an independent prognostic predictor of ACLF, which has not been reported in the literature.

This study has some limitations. First, our sample size was relatively small. Moreover, the present study was a single-center investigation in China. The findings need to be confirmed in large multicenter and prospective studies. Overall, our findings demonstrate that IL-6 is preferentially enriched in patients with HBV-ACLF and that this increase positively correlates with disease severity. Furthermore, our data indicate that an increased serum level of IL- 6 could be an independent predictor of mortality in patients with HBVACLF.

\section{Abbreviations}

HBV-ACLF: hepatitis B virus (HBV)-related acute-on-chronic liver failure; ROC: the receiver operating characteristic; MELD: the Model for End-Stage Liver Disease; SIRS: systemic inflammatory response; PTA: prothrombin time activity; MELD: model for end-stage liver disease; $\mathrm{Cr}$, creatinine; $\mathrm{PCT}$ : procalcitonin.CHB: chronic hepatitis B; NC: normal controls; IL-1a:interleukin-1 alpha; EGF: epidermal growth factor; G-CSF: granulocyte colony-stimulating factor; GM-CSF: granulocyte macrophage colony-stimulating factor; IFNa2: interferon alpha-2; IP-10: interferon gamma-induced protein 10; MCP-1: monocyte chemotactic protein-1; TNFa:tumor necrosis factor alpha; VEGF: vascular endothelial growth factor; FGF-2: fibroblast growth factor 2; TGF-a: transforming growth factor alpha; FIT-3L:Flt-3 ligand; GRO: growth-regulated oncogene; MDC:macrophage-derived chemokine; sCD40L: soluble CD40 ligand; CXCL9:chemokine (C-X-C motif) ligand 9; ALT: alanine aminotransferase; AST: aspartate aminotransferase; PTA: prothrombin time activity; TBil: total bilirubin; SBP: spontaneous bacterial peritonitis; UGB: upper gastrointestinal bleeding; MELD: model for end-stage liver disease; ULN: upper limit of normal; N.D: not determined.

AUC, area under curve; ROC curve, receiver operating characteristic curve; AUROC, area under the ROC curve; Cum, cumulative.

\section{Declarations}

\section{Ethics approval and consent to participate}

All procedures performed in studies involving human participants were in accordance with the ethical standards of the institutional and/or national research committee, and with the guidelines of the Declaration of Helsinki and its later amendments or comparable ethical standards. Informed consent was obtained from all individual participants included in the study.

\section{Consent for publication}

Not applicable. 


\section{Competing interests}

The authors declare that no conflict of interest exists.

\section{Funding}

This study was supported by grants from the National major science and technology project for the prevention and treatment of AIDS and viral hepatitis (2018ZX10302204-002, 2018ZX10302205-002), Natural Science Foundation of China (No.82070611, 81873572 and 81901942), Guangzhou Science and Technology Plan Projects (201904010442), Sun Yat-Sen University Clinical Research 5010 Program (2020007 and 2018009), Research project on degree and postgraduate education reform in guangdong province (2018JGXM04), Young teacher training program of sun yat-sen university (16ykpy40), and the Five-Year Plan of Third Affiliated Hospital of Sun Yat-sen University (K00006).

\section{Authors' contributions}

Design of the study: YYZ, GLZ. Acquisition of data: YYZ, LNW, LW, JX, XJL and JGL. Data analysis: YYZ, GLZ. Manuscript preparation: YYZ, GLZ, CX and LP. All authors reviewed the manuscript and gave final approval for the work.

\section{Acknowledgements}

The authors would like to thank the patients, the healthy volunteers and all investigators involved in this study.

\section{Availability of data and material}

All data generated or analyzed during this study are included in this article.

\section{References}

1. Sarin SK, Choudhury A: Acute-on-chronic liver failure: terminology, mechanisms and management. Nat Rev Gastroenterol Hepatol 2016, 13:131-149.

2. Wang FS, Zhang Z: Liver: How can acute-on-chronic liver failure be accurately identified? Nat Rev Gastroenterol Hepatol 2013, 10:390-391.

3. Arroyo V, Moreau R, Jalan R: Acute-on-Chronic Liver Failure. N Engl J Med 2020, 382:2137-2145.

4. Hernaez R, Solà E, Moreau R, Ginès P: Acute-on-chronic liver failure: an update. Gut 2017, 66:541553.

5. Clària J, Stauber RE, Coenraad MJ, Moreau R, Jalan R, Pavesi M, Amorós À, Titos E, Alcaraz-Quiles J, Oettl $\mathrm{K}$, et al: Systemic inflammation in decompensated cirrhosis: Characterization and role in acuteon-chronic liver failure. Hepatology 2016, 64:1249-1264. 
6. Wang K, Wu ZB, Ye YN, Liu J, Zhang GL, Su YJ, He HL, Zheng YB, Gao ZL: Plasma Interleukin-10: A Likely Predictive Marker for Hepatitis B Virus-Related Acute-on-Chronic Liver Failure. Hepat Mon 2014, 14:e19370.

7. Zhang GL, Zhao QY, Xie C, Peng L, Zhang T, Gao ZL: Elevated Serum IgG Levels Positively Correlated with IL-27 May Indicate Poor Outcome in Patients with HBV-Related Acute-On-Chronic Liver Failure. J Immunol Res 2019, 2019:1538439.

8. Sole C, Sola E, Morales-Ruiz M, Fernandez G, Huelin P, Graupera I, Moreira R, de Prada G, Ariza X, Pose E, et al: Characterization of Inflammatory Response in Acute-on-Chronic Liver Failure and Relationship with Prognosis. Sci Rep 2016, 6:32341.

9. Bao S, Zheng J, Li N, Huang C, Chen M, Cheng Q, Li Q, Lu Q, Zhu M, Ling Q, et al: Role of interleukin23 in monocyte-derived dendritic cells of HBV-related acute-on-chronic liver failure and its correlation with the severity of liver damage. Clin Res Hepatol Gastroenterol 2017, 41:147-155.

10. Schmidt-Arras D, Rose-John S: IL-6 pathway in the liver: From physiopathology to therapy. J Hepatol 2016, 64:1403-1415.

11. Naseem S, Hussain T, Manzoor S: Interleukin-6: A promising cytokine to support liver regeneration and adaptive immunity in liver pathologies. Cytokine Growth Factor Rev 2018, 39:36-45.

12. Loosen SH, Schulze-Hagen M, Leyh C, Benz F, Vucur M, Kuhl C, Trautwein C, Tacke F, Bruners P, Roderburg C, Luedde T: IL-6 and IL-8 Serum Levels Predict Tumor Response and Overall Survival after TACE for Primary and Secondary Hepatic Malignancies. Int J Mol Sci 2018, 19.

13. Liu F, Li L, Xu M, Wu J, Luo D, Zhu Y, Li B, Song X, Zhou X: Prognostic value of interleukin-6, C-reactive protein, and procalcitonin in patients with COVID-19. J Clin Virol 2020, 127:104370.

14. Lin S, Huang Z, Wang M, Weng Z, Zeng D, Zhang Y, Zhu Y, Jiang J: Interleukin-6 as an early diagnostic marker for bacterial sepsis in patients with liver cirrhosis. J Crit Care 2015, 30:732-738.

15. Labenz C, Toenges G, Huber Y, Nagel M, Marquardt JU, Schattenberg JM, Galle PR, Labenz J, Worns MA: Raised serum Interleukin-6 identifies patients with liver cirrhosis at high risk for overt hepatic encephalopathy. Aliment Pharmacol Ther 2019, 50:1112-1119.

16. Jekarl DW, Lee SY, Lee J, Park YJ, Kim Y, Park JH, Wee JH, Choi SP: Procalcitonin as a diagnostic marker and IL-6 as a prognostic marker for sepsis. Diagn Microbiol Infect Dis 2013, 75:342-347.

17. Lai HS, Lin WH, Lai SL, Lin HY, Hsu WM, Chou CH, Lee PH: Interleukin-6 mediates angiotensinogen gene expression during liver regeneration. PLoS One 2013, 8:e67868.

18. Taniguchi K, Karin M: IL-6 and related cytokines as the critical lynchpins between inflammation and cancer. Semin Immunol 2014, 26:54-74.

19. Dirchwolf M, Podhorzer A, Marino M, Shulman C, Cartier M, Zunino M, Paz S, Munoz A, Bocassi A, Gimenez J, et al: Immune dysfunction in cirrhosis: Distinct cytokines phenotypes according to cirrhosis severity. Cytokine 2016, 77:14-25.

\section{Tables}


Table 1: Clinical characteristics of the participants enrolled in the study

\begin{tabular}{|c|c|c|c|}
\hline Group & HBV-ACLF $(n=40)$ & $\mathrm{CHB}(\mathrm{n}=30)$ & $N C(n=25)$ \\
\hline Gender(male) & 37 & 20 & 18 \\
\hline Age(years) & $42.88 \pm 9.31$ & $40.17 \pm 12.18$ & $41.08 \pm 12.18$ \\
\hline WBC $\left(10^{9} / \mathrm{L}\right)$ & $6.56 \pm 3.45$ & $5.97 \pm 1.43$ & $6.19 \pm 1.34$ \\
\hline $\operatorname{PLT}\left(10^{9} / \mathrm{L}\right)$ & $99.53 \pm 67.44$ & $208.58 \pm 65.69$ & $242.96 \pm 37.81$ \\
\hline $\operatorname{ALT}(\mathrm{U} / \mathrm{L})$ & $201.9 \pm 254.42$ & $32.07 \pm 27.56$ & $19.17 \pm 12.46$ \\
\hline AST (U/L) & $215.65 \pm 532.74$ & $26.52 \pm 10.79$ & $19.35 \pm 5.45$ \\
\hline PTA (\%) & $33.28 \pm 7.86$ & N.D. & N.D. \\
\hline INR (\%) & $2.53 \pm 0.89$ & N.D. & N.D. \\
\hline Albumin (g/L) & $37.45 \pm 4.39$ & $48.26 \pm 2.99$ & N.D. \\
\hline TBiL(umol/L) & $349.97 \pm 204.92$ & $12.10 \pm 6.09$ & N.D. \\
\hline CR(umol/L) & $77.18 \pm 40.65$ & $70.83 \pm 16.02$ & $70.80 \pm 13.52$ \\
\hline $\mathrm{Na}$ (umol/L) & $136.20 \pm 5.68$ & N.D. & N.D. \\
\hline HBeAg positive & 9 & 8 & 0 \\
\hline $\begin{array}{l}\text { HBV-DNA } \\
(\log 10 \mathrm{lU} / \mathrm{ml})\end{array}$ & $4.34 \pm 1.63$ & $3.80 \pm 1.01$ & 0 \\
\hline Complication & 43 & 0 & 0 \\
\hline Ascites & 14 & 0 & 0 \\
\hline SBP & 13 & 0 & 0 \\
\hline Hepatic encephalopathy & 10 & 0 & 0 \\
\hline Hepatorenal syndrome & 3 & 0 & 0 \\
\hline UGB & 3 & 0 & 0 \\
\hline MELD score & $24.79 \pm 6.62$ & N.D. & N.D. \\
\hline MELD-Na score & $22.88 \pm 12.32$ & N.D. & N.D. \\
\hline
\end{tabular}

Note: Data are shown as means \pm SD. ACLF: acute-on-chronic liver failure; HC: healthy control; ALT: alanine aminotransferase; AST: aspartate aminotransferase; PTA: prothrombin time activity; TBil: total bilirubin; SBP: spontaneous bacterial peritonitis; UGB: upper gastrointestinal bleeding; MELD: model for end-stage liver disease; ULN: upper limit of normal; N.D: not determined 
Table 2. ROC curve analysis of IL-6, CXCL9, IL-15 and MDC.

\begin{tabular}{|llllll|}
\hline Indicators & Specificity & Sensitivity & AUC $(95 \% \mathrm{Cl})$ & P value & Cut-off \\
\hline IL-6 & 0.73 & 0.64 & $0.703(0.507-0.899)$ & 0.034 & $8.285 \mathrm{pg} / \mathrm{ml}$ \\
\hline CXCL9 & 0.73 & 0.72 & $0.691(0.510-0.871)$ & 0.046 & $2982.5 \mathrm{pg} / \mathrm{ml}$ \\
\hline IL-15 & 0.53 & 0.84 & $0.637(0.437-0.838)$ & 0.150 & $14.21 \mathrm{pg} / \mathrm{ml}$ \\
\hline MDC & 0.27 & 0.56 & $0.285(0.115-0.456)$ & 0.025 & $492.735 \mathrm{pg} / \mathrm{ml}$ \\
\hline MELD & 0.73 & 0.84 & $0.765(0.579-0.951)$ & 0.005 & 26.50 \\
\hline MELD-Na & 0.73 & 0.80 & $0.779(0.612-0.945)$ & 0.004 & 25.13 \\
\hline
\end{tabular}

Note: Data in parentheses are $95 \%$ confidence interval.

Abbreviations: AUC, area under curve; ROC curve, receiver operating characteristic curve.

Table 3. Characteristics of patients with HBV-ACLF according to clinical outcome 


\begin{tabular}{|llll|}
\hline Group & $\begin{array}{l}\text { Non-surviving } \\
(\mathrm{n}=15)\end{array}$ & $\begin{array}{l}\text { Surviving } \\
(\mathrm{n}=25)\end{array}$ & P-value \\
\hline Gender(male) & 14 & 23 & $\mathrm{~ns}$ \\
\hline Age(years) & $46.07(28-63)$ & $41.80(26-57)$ & $\mathrm{ns}$ \\
\hline Cirrhosis & 12 & 17 & $\mathrm{~ns}$ \\
\hline WBC(109/L) & $7.98(1.95-16.96)$ & $5.49(1.22-10.41)$ & 0.019 \\
\hline PLT(109/L) & $64.8(14-166)$ & $116.32(19-280)$ & 0.0162 \\
\hline ALT (U/L) & $346.6(32-3376)$ & $133.76(39-935)$ & $n s$ \\
\hline AST (U/L) & $190.27(23-1096)$ & $133.76(39-935)$ & $n s$ \\
\hline PTA (\%) & $28.43(14-41)$ & $35.52(27-49)$ & 0.0072 \\
\hline INR (\%) & $3.14(1.85-5.92)$ & $2.22(1.65-2.75)$ & 0.0013 \\
\hline Albumin (g/L) & $36.57(31.2-41.7)$ & $37.9(29.8-52.6)$ & $n s$ \\
\hline TBiL(umol/L) & $402.02(36.5-747.5)$ & $321.86(91-681)$ & $n s$ \\
\hline CR(umol/L) & $95.27(33-250)$ & $67.01(42-95.4)$ & 0.0312 \\
\hline Na(umol/L) & $133.34(113.8-143)$ & $137.64(130-146)$ & 0.0225 \\
\hline PCT & $2.24(0.3-12.12)$ & $0.72(0.13-1.49)$ & 0.0476 \\
\hline MELD score & $28.54 \pm 8.77$ & $22.86 \pm 3.9$ & 0.0076 \\
\hline MELD-Na score & $31.18 \pm 15.48$ & $18.67 \pm 7.92$ & 0.0016 \\
\hline IL-6(pg/ml) & $25.84(1.07-15.10)$ & $7.67(1.53-15.10)$ & 0.0068 \\
\hline IL-15(pg/ml) & $27.46(4.76-100.07)$ & $12.41(5.03-53.51)$ & 0.0218 \\
\hline MDC(pg/ml) & $351.39(58.8-810.4)$ & $485.92(157.29-1086)$ & 0.0397 \\
\hline CXCL9(pg/ml) & 5930.07 & 3140.53 & 0.0364 \\
\hline & $(855.07-22903)$ & $(871.27-9418)$ & \\
\hline
\end{tabular}

Note: Data are shown as mean and standard deviations or medians and ranges.

Abbreviations: ACLF, acute-on-chronic liver failure; ALT: alanine aminotransferase; PLT, platelet counts; AST: aspartate aminotransferase; PTA: prothrombin time activity; TBil: total bilirubin; MELD: model for end-stage liver disease; ALB, albumin; ALT, alanine aminotransferase; AST, aspartate aminotransferase; $\mathrm{Cr}$, creatinine; MELD, model for end-stage liver disease;

Table 4. Factors associated with mortality in patients with HBV-ACLF by using the Cox regression analysis 


\begin{tabular}{|c|c|c|c|c|}
\hline Parameters & Wald & P-value & $\mathrm{HR}$ & $95 \% \mathrm{Cl}$ \\
\hline \multicolumn{5}{|c|}{ Univariate analysis } \\
\hline IL-6(pg/ml) & 12.166 & $<0.001$ & 1.031 & $1.014-1.049$ \\
\hline IL-15(pg/ml) & 5.103 & 0.024 & 1.019 & $1.003-1.036$ \\
\hline $\mathrm{MDC}(\mathrm{pg} / \mathrm{ml})$ & 3.475 & 0.062 & 0.997 & $0.994-1.000$ \\
\hline CXCL9(pg/ml) & 4.580 & 0.032 & 1.000 & $1.000-1.000$ \\
\hline WBC $\left(10^{9} / \mathrm{L}\right)$ & 9.999 & 0.002 & 1.323 & $1.112-1.574$ \\
\hline $\operatorname{PLT}\left(10^{9} / \mathrm{L}\right)$ & 4.864 & 0.027 & 0.987 & $0.976-0.999$ \\
\hline PTA(\%) & 8.170 & 0.004 & 0.892 & $0.824-0.965$ \\
\hline INR & 14.753 & $<0.001$ & 2.705 & $1.628-4.494$ \\
\hline $\mathrm{Na}$ (ummol/L) & 6.936 & 0.008 & 0.897 & $0.827-0.972$ \\
\hline CR(ummol/L) & 4.313 & 0.038 & 1.009 & $1.000-1.017$ \\
\hline PCT & 3.152 & 0.076 & 1.139 & $0.987-1.315$ \\
\hline MELD & 9.352 & 0.002 & 1.144 & $1.050-1.247$ \\
\hline MELD-Na & 11.950 & 0.001 & 1.068 & $1.029-1.109$ \\
\hline \multicolumn{5}{|c|}{ Multivariate analysis } \\
\hline IL-6(pg/ml) & 5.051 & 0.025 & 1.026 & $1.026-1.003$ \\
\hline WBC $\left(10^{9} / L\right)$ & 11.490 & 0.001 & 1.344 & $1.344-1.133$ \\
\hline $\operatorname{PLT}\left(10^{9} / \mathrm{L}\right)$ & 6.848 & 0.009 & 0.979 & $0.979-0.964$ \\
\hline
\end{tabular}

Note: Data are shown as mean and standard deviations or medians and ranges.

Abbreviations: PTA: prothrombin time activity; MELD: model for end-stage liver disease; $\mathrm{Cr}$, creatinine; PCT: procalcitonin.

\section{Figures}




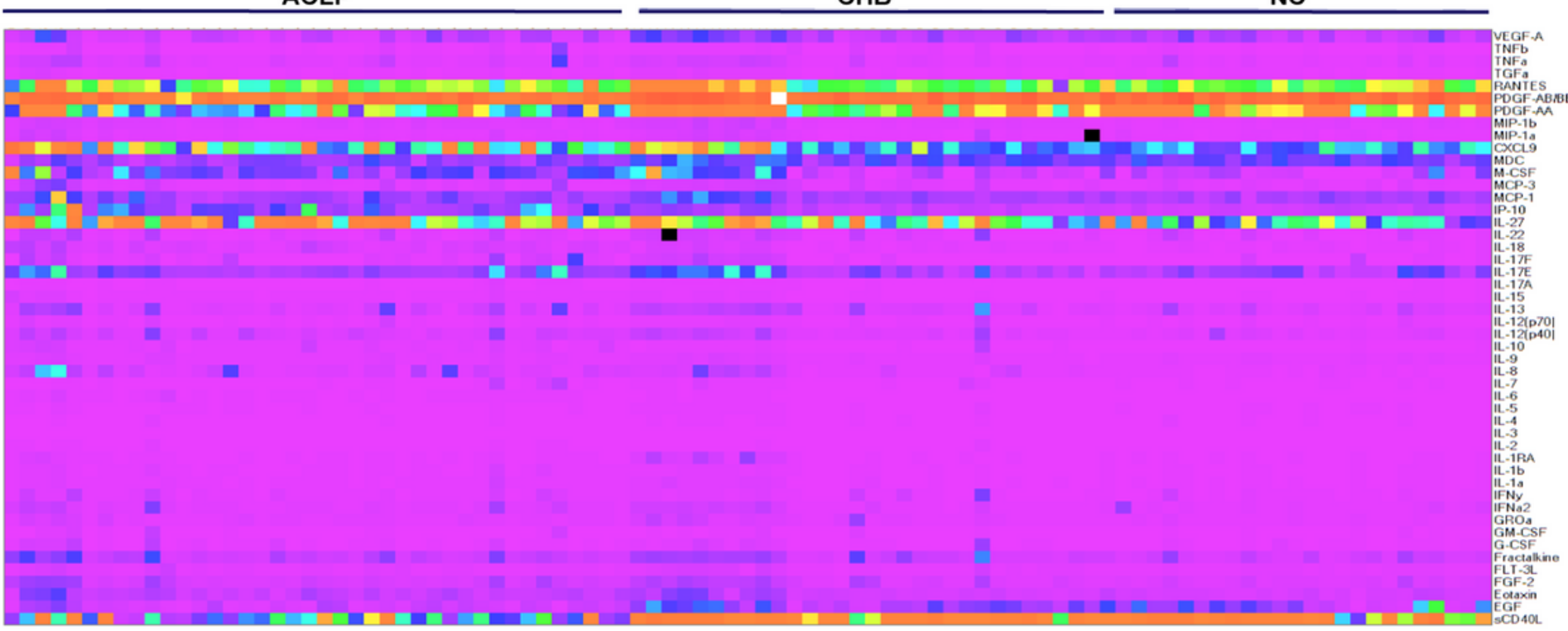

B
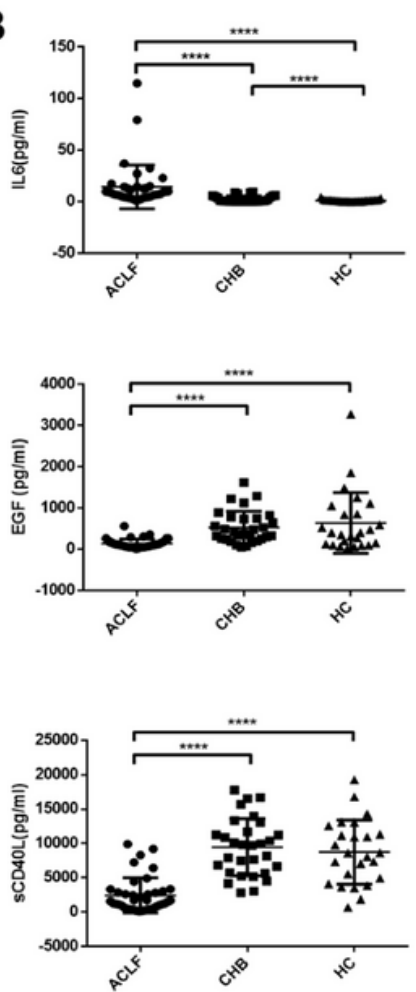
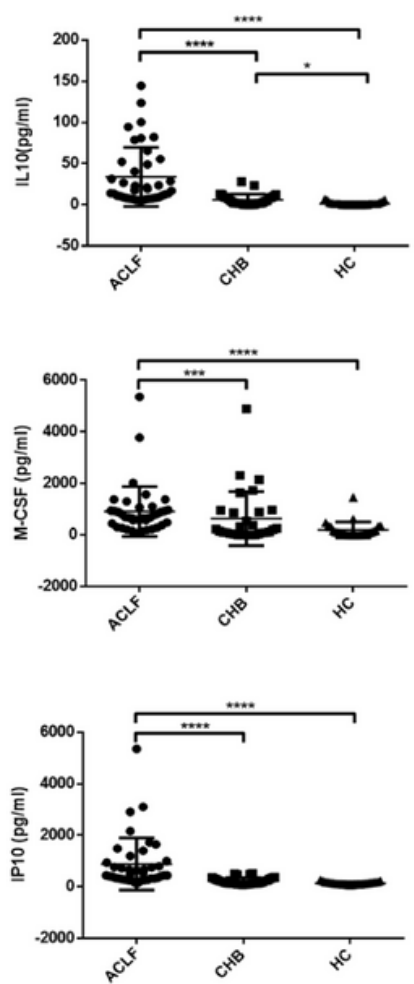
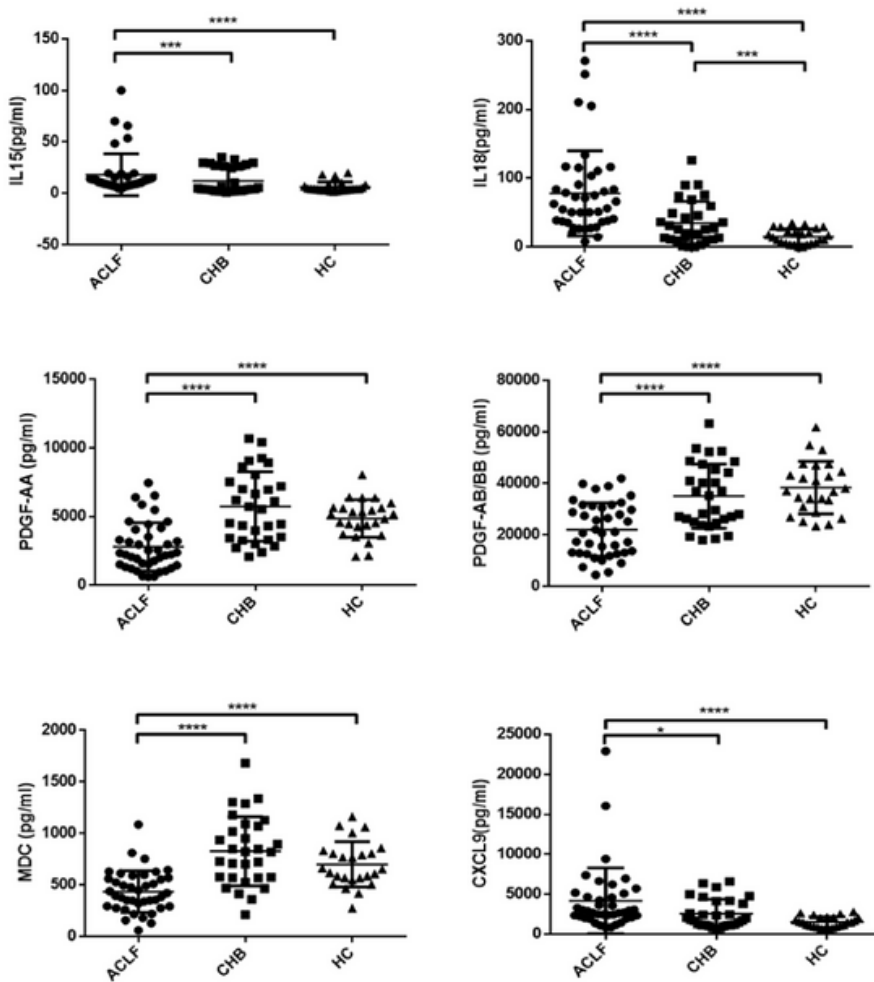

Figure 1

Twelve cytokines were significantly different in patients with HBV-ACLF from other groups. A. Cluster heat map of 48 cytokines detected by luminex among three groups. B. The level of IL-6, IL-10, IL-15, IL-18, MCSF, IP-10 and CXCL9 were significantly higher in patients with HBV-ACLF than in either patients with CHB or NC subjects. While the level of EGF, PDGF-AA, PDGF-AB/BB, MDC and sCD40L were significantly lower. ${ }^{*} \mathrm{P}<0.05 ;{ }^{*} \mathrm{P}<0.01 ; * \star * \mathrm{P}<0.001 ; * \star \star \star * P<0.0001$. 
A

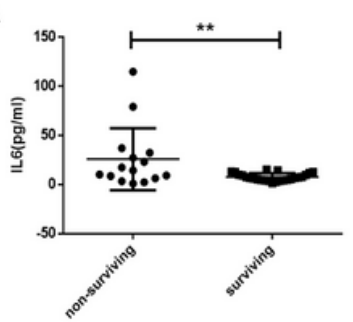

B
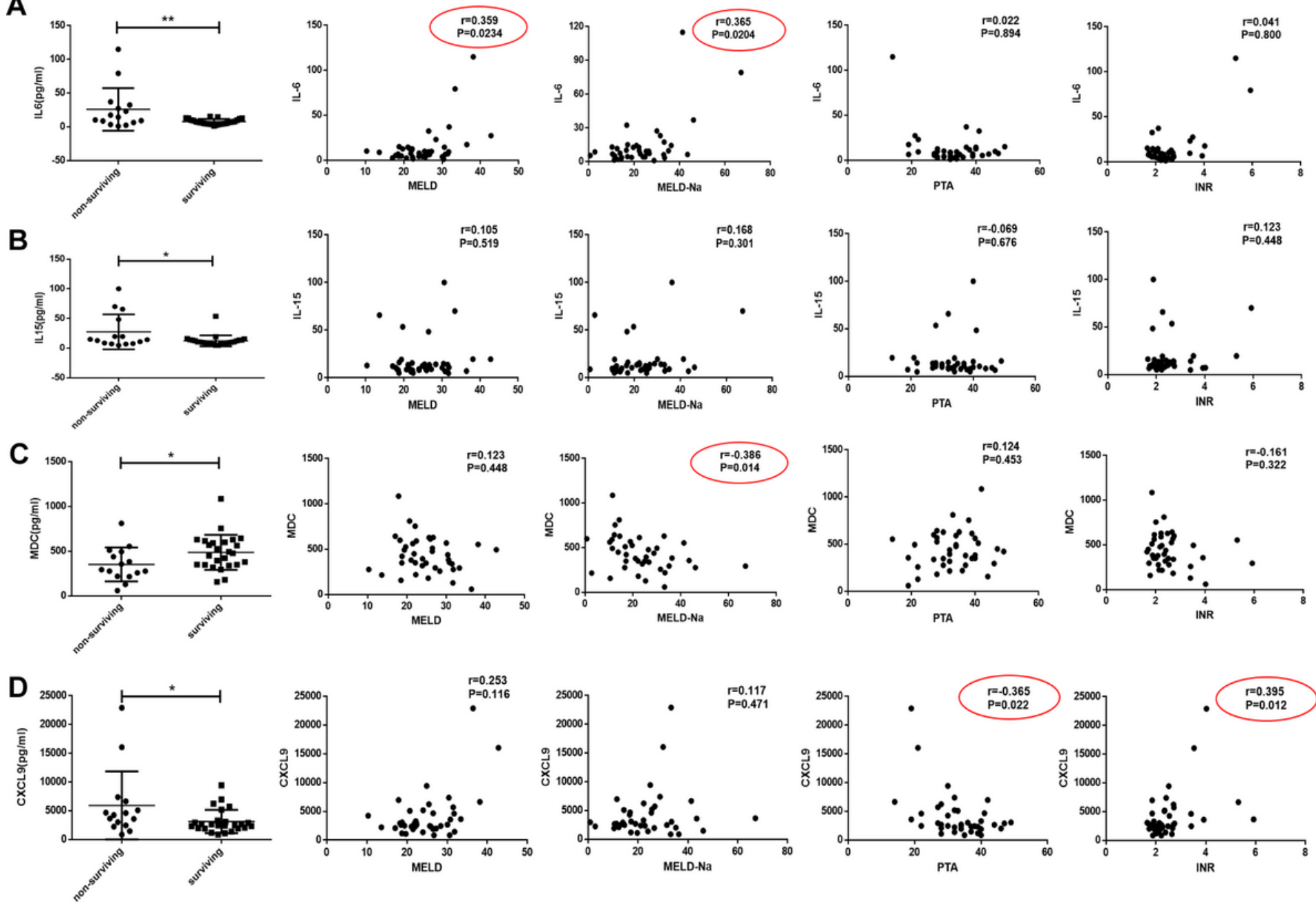

Figure 2

Four out of twelve cytokines were related to clinical outcome including IL-6, IL-15, CXCL9 and MDC. Notes: A. The serum level of IL-6 was significantly higher in non-surviving patients than in surviving patients $(P<0.01)$. Moreover, the concentrations of IL6 were positively correlated with MELD, MELD-Na scores $(P<0.05)$. B. The serum level of IL-15 was higher in non-surviving patients than in surviving patients $(P<0.05)$, while the concentrations of IL-15 had no correlation with MELD, MELD-Na scores, PTA and INR. C. The serum level of MDC was higher in non-surviving patients than in surviving patients $(P<0.05)$. In addition, the concentrations of MDC were negatively correlated with MELD-Na scores $(P<0.05)$. D. The serum level of CXCL9 was higher in non-surviving patients than in surviving patients $(P<0.05)$. 
A

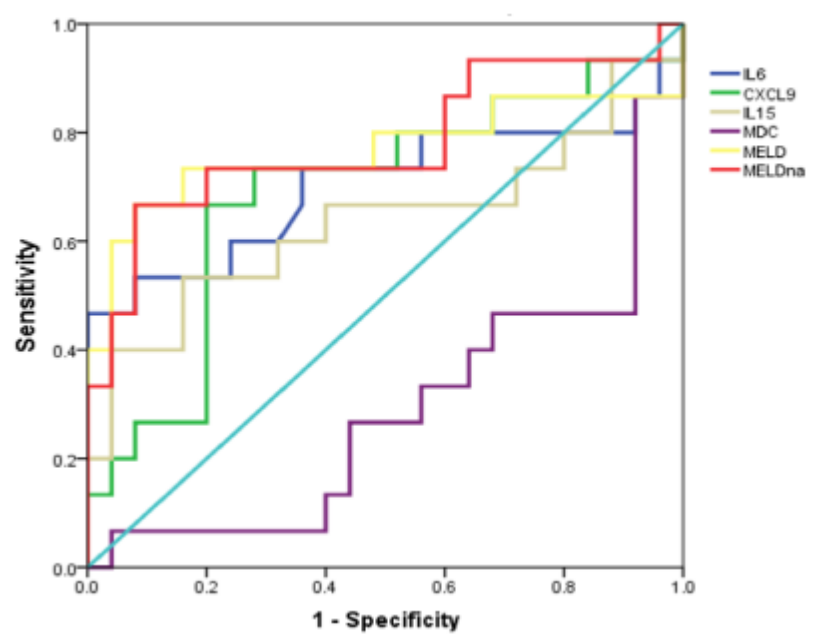

B
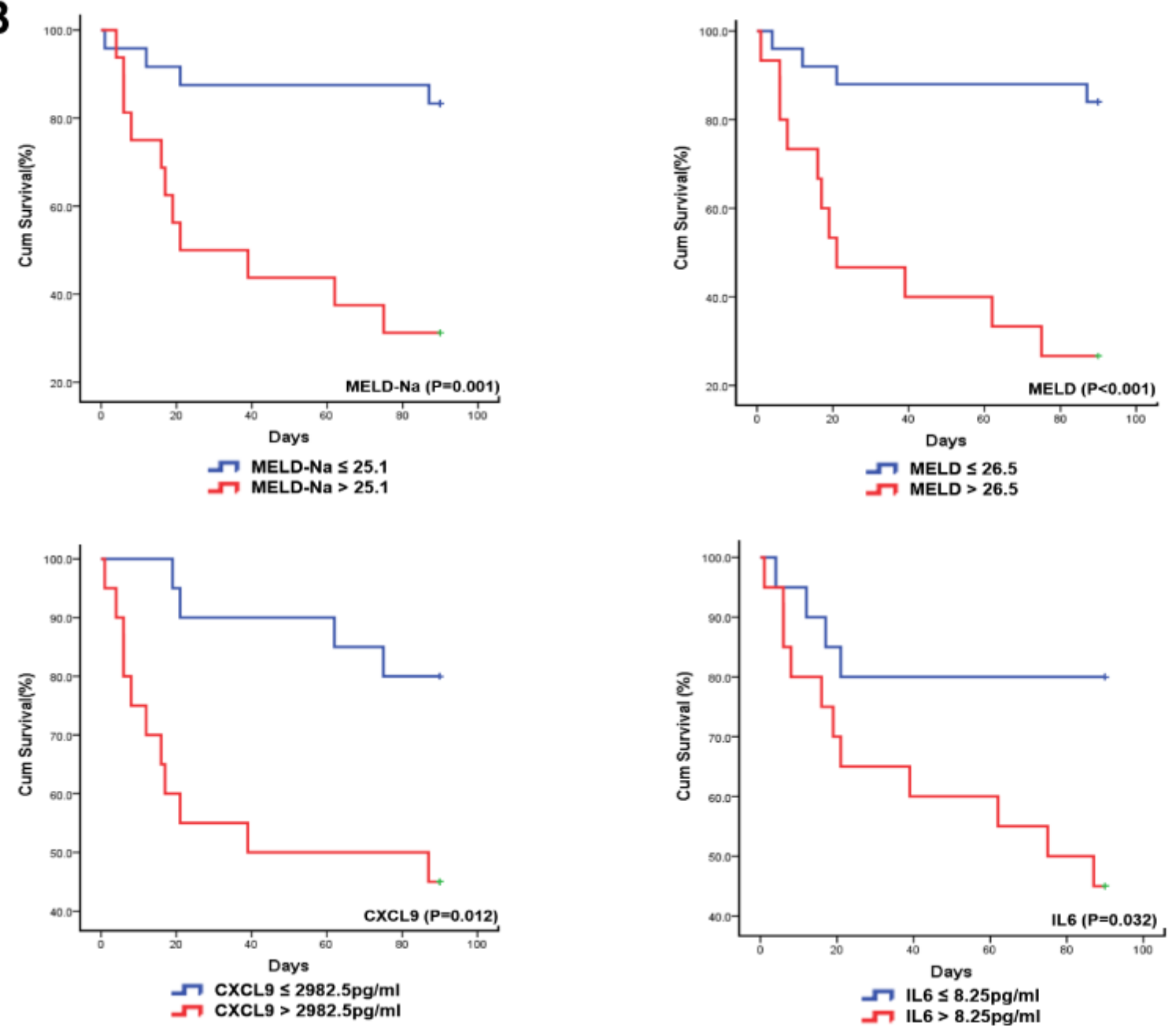

\section{Figure 3}

Increased serum IL-6 and CXCL9 indicated poor prognosis in HBV-ACLF. Notes: (A) The prognostic value of IL-6, IL-15, MDC and CXCL9 were assessed by the ROC curve analysis, compared with MELD and MELD-Na scores. Specific analysis results were shown in Table2. (B) Survival was evaluated using Kaplan-Meier curves, and the statistics were compared by log-rank tests. Significant differences between 
the higher group and the lower group in 90-day survival rate (IL6, $P=0.032 ; C X C L 9, P=0.012 ; M E L D$, $\mathrm{P}=0.001$; MELD-Na, $\mathrm{P}<0.001$; Figure 3B). 